\title{
Analysis of bias stress on thin-film transistors obtained by Hot-Wire Chemical Vapour Deposition
}

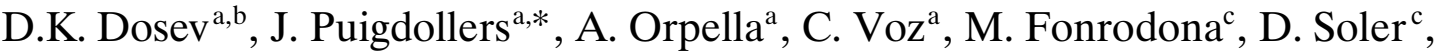 \\ L.F. Marsal ${ }^{\mathrm{b}}$, J. Pallarès ${ }^{\mathrm{b}}$, J. Bertomeu $^{\mathrm{c}}$, J. Andreu $^{\mathrm{c}}$, R. Alcubilla ${ }^{\mathrm{a}}$ \\ ${ }^{a}$ Department Enginyeria Electrònica, Universitat Politècnica de Catalunya, Barcelona, Spain \\ ${ }^{\mathrm{b}}$ Department d'Enginyeria Electrònica, Elèctrica i Automàtica, U. Rovira i Virgili, Tarragona, Spain \\ ${ }^{\mathrm{c}}$ Department de Física Aplicada i Òptica, Universitat de Barcelona, Barcelona, Spain
}

\begin{abstract}
The stability under gate bias stress of unpassivated thin film transistors was studied by measuring the transfer and output characteristics at different temperatures. The active layer of these devices consisted of in nanocrystalline silicon deposited at $125^{\circ} \mathrm{C}$ by Hot-Wire Chemical Vapour Deposition. The dependence of the subthreshold activation energy on gate bias for different gate bias stresses is quite different from the one reported for hydrogenated amorphous silicon. This behaviour has been related to trapped charge in the active layer of the thin film transistor. (c) 2001 Elsevier Science B.V. All rights reserved.
\end{abstract}

Keywords: TFT; Nanocrystalline silicon; HWCVD; Stability

\section{Introduction}

Hot-Wire Chemical Vapour Deposition (HWCVD) is one of the most promising techniques to obtain hydrogenated amorphous silicon (a-Si:H) and hydrogenated nanocrystalline silicon (nc-Si:H) at low substrate temperatures $\left(<300^{\circ} \mathrm{C}\right)$ over large areas and high deposition rates $(>1 \mathrm{~nm} / \mathrm{s})[1-3]$

Hydrogenated nanocrystalline silicon obtained by HWCVD is composed of small silicon crystallites, with an average grain size of a few nanometers embedded in an amorphous silicon matrix. As a result, nc-Si:H properties lay between those of a-Si:H and polysilicon (with grain sizes of few micrometers).

Hydrogenated amorphous silicon thin-film transistors (TFT) are widely used in liquid crystal displays and matrix image sensors. However a-Si:H TFTs present electrical instability, mainly consisting of a threshold

\footnotetext{
*Corresponding author. Tel. +34-93-4011002; fax: +34-934016756.

E-mail address: jpuigd@eel.upc.es (J. Puigdollers).
}

voltage $\left(V_{\mathrm{t}}\right)$ shift, when a gate voltage is applied for a prolonged period of time. Two mechanisms contribute to threshold voltage instability: charge trapping in the gate insulator and defect creation in the band-gap of a-Si:H [4].

In this paper, the stability of TFTs with nc-Si:H active layers deposited at $125^{\circ} \mathrm{C}$ by HWCVD is studied. The stability was investigated by examining the gate transfer characteristics and the dependence of the subthreshold current activation energy on gate bias for as-deposited samples and after negative and positive bias stress.

\section{Experimental}

Nanocrystalline thin-film transistors were deposited by HWCVD in an ultra-high-vacuum multichamber set-up described elsewhere [5]. Process gases [silanehydrogen mixture $(4 / 76 \mathrm{sccm})]$ were catalytically dissociated by a tungsten wire heated to $1700^{\circ} \mathrm{C}$. Substrate temperature was around $125^{\circ} \mathrm{C}$ (measured by a thermocouple attached to the heater) and the process pressure 
was $1 \times 10^{-2}$ mbar. Under these conditions the deposition rate was $0.6 \mathrm{~nm} / \mathrm{s}$. Thin-film transistors used in this work are inverted staggered structures without top passivation layer. A thermally oxidised n-type (100) silicon wafer with a resistivity of $1-10 \Omega / \mathrm{cm}$ was used as substrate. Thicknesses were $250 \mathrm{~nm}$ for the active layer and $250 \mathrm{~nm}$ for the silicon dioxide. An $\mathrm{n}^{+}$-doped layer $(50 \mathrm{~nm})$ between the drain and source contacts and the intrinsic layer was also deposited by HWCVD. Finally a chromium layer was thermally evaporated and the metallic contacts (drain and source) were delimited by photolithographic techniques. The channel width was $137 \mu \mathrm{m}$ and its length $55 \mu \mathrm{m}$, giving an aspect ratio for the device (i.e. $\mathrm{W} / \mathrm{L}$ ) of 2.5. Details on the fabrication process have been reported in a previous work [6].

Different TFTs were electrically characterised by measuring the transfer and output characteristics. We also studied the dependence of the subthreshold current activation energies $\left(E_{\mathrm{ACT}}=E_{\mathrm{C}}-E_{\mathrm{F}}\right)$ on gate bias. After the initial characterisation of the samples, stressing processes at different gate voltages $(-40,-20$ and $+40 \mathrm{~V})$ were performed at room temperature $(298 \mathrm{~K})$ for $1000 \mathrm{~s}$. All the measurements were performed under dark conditions by using a Hewlett-Packard HP4145B semiconductor parameter analyser and a temperature controlled chuck.

\section{Results and discussion}

The output and transfer characteristics of the transistors as deposited and after positive and negative gate bias stress are shown in Fig. 1a. From the output characteristics $I_{\mathrm{DS}}$ vs. $V_{\mathrm{DS}}$ it can be seen that there is not a current crowding and as a consequence we conclude that the influence of the source and drain contact resistance is not important in our devices. The absence of the Kink effect (a rapid rise of $I_{\mathrm{DS}}$ at high $V_{\mathrm{DS}}$ ) indicates that the electron injection at the contacts is very small. The transfer characteristics in saturation regime were measured to determine the threshold voltage and the field effect mobility of the transistors. A threshold voltage of $6.9 \mathrm{~V}$ and field effect mobility of $0.3 \mathrm{~cm}^{2} / \mathrm{V} \cdot \mathrm{s}$ were obtained. After gate bias stress, a positive shift of threshold voltage $\left(\Delta V_{\mathrm{t}} \approx+1 \mathrm{~V}\right)$ for positive gate bias stress and a negative shift $\left(\Delta V_{\mathrm{t}} \approx-1\right.$ $\mathrm{V})$ for negative gate bias stress were obtained.

The application of a gate voltage in field effect structures shifts the Fermi level inside the gap. This displacement allows us to study the electronic properties of the material. The rate at which $E_{\mathrm{F}}$ moves towards the conduction band (in n-channel devices) depends on the density of states located in the band gap and on the distribution of tail states close to the conduction band. For small values of $V_{\mathrm{GS}}$ the Fermi level is located in deep states. Increasing $V_{\mathrm{GS}}$ shifts the Fermi level to the conduction band, and the tail band states become important. Besides, for high positive $V_{\mathrm{GS}}$ applied an influence of the amorphous phase in the nucleation region of the crystallites could not be discarded.

It has been reported (experimentally as well as through simulation results) $[7,8]$ that for hydrogenated amorphous silicon, the application of a negative gate bias create additional states in the upper half part of the bandgap. This additional peak on the density of states leads to a reduction of the slope of $E_{\mathrm{ACT}}\left(V_{\mathrm{GS}}\right)$ for $V_{\mathrm{GS}}<10 \mathrm{~V}$, indicating the presence of defect states created by degradation.

In Fig. 2 the dependence of $E_{\mathrm{ACT}}$ on $V_{\mathrm{GS}}$ for TFTs before and after bias stress degradation is shown.

For the as-deposited sample we observe that the Fermi level moves from around midgap $\left(E_{\mathrm{ACT}}=0.45\right.$ $\mathrm{eV}$ for $\left.V_{\mathrm{GS}}=0 \mathrm{~V}\right)$ to the conduction band, remaining nearly constant at approximately $0.15 \mathrm{eV}$, where the Fermi level is pinned by the band tail states.

In Fig. 2 it is observed that the application of positive (negative) bias stress to the gate leads to a positive (negative) displacement of the $\left[E_{\mathrm{ACT}}\left(V_{\mathrm{GS}}\right)\right]$ characteris-

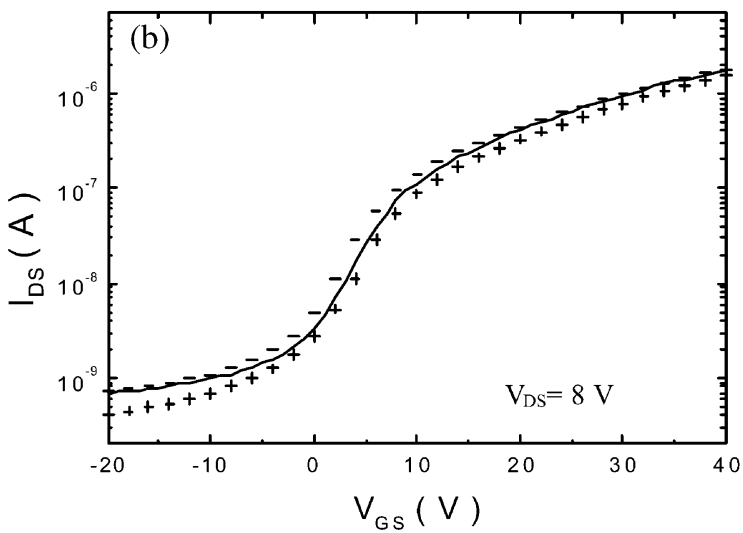

Fig. 1. Output and transfer characteristics of nanocrystalline TFT measured as deposited (solid lines) and after different gate bias stress: $+40 \mathrm{~V}$ (+ symbols), $-40 \mathrm{~V}$ ( - symbols). 


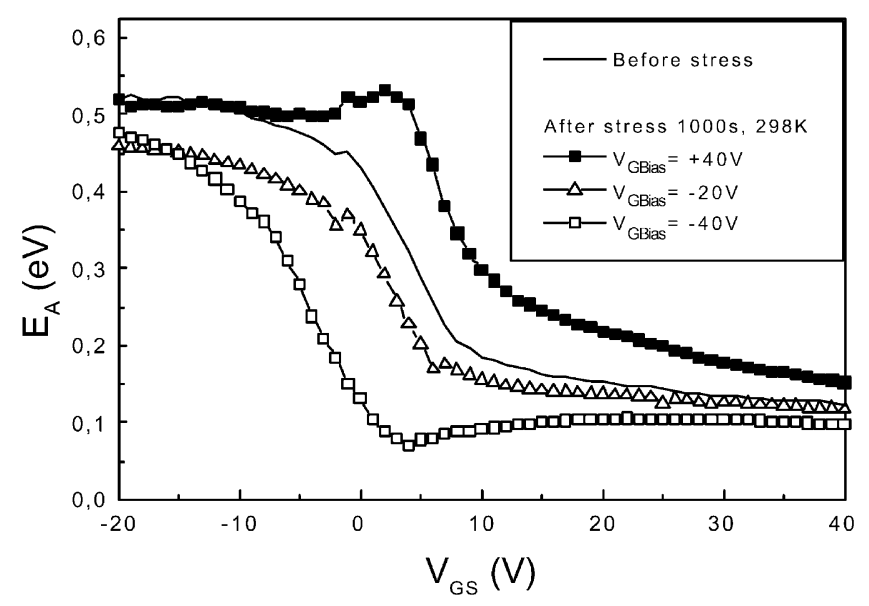

Fig. 2. Dependence of the activation energy $\left(E_{\mathrm{ACT}}=E_{\mathrm{C}}-E_{\mathrm{F}}\right)$ of the subthreshold transfer characteristics for TFT stressed at different gate bias.

tics. The shift obtained after the application of negative gate bias stress is in opposite direction to the one observed for a-Si:H TFTs after a similar degradation process [8].

We believe that the application of positive/negative bias stress to the gate causes redistributions in the trapped charge of the active layer in the TFT. Negative stress, for example, depletes the trapped charge between $E_{\mathrm{F}}$ and the conduction band, causing the Fermi level to be slightly shifted towards the conduction band. Therefore, the application of small values of $V_{\mathrm{GS}}$ easily shifts the $E_{\mathrm{F}}$ to the conduction band, remaining fixed by the band-tail states. This charge trapped could be related to the impurities (oxygen, copper,...) incorporated in the layer during the deposition process [9].

The transistors were measured again $50 \mathrm{~h}$ after the stressing process. They showed the same characteristics as before the stressing process including threshold voltage and activation energy, these results also seem to confirm that charge trapping could be the main mechanism involved by the application of gate bias stress.

\section{Conclusions}

In this work we have presented the results of stress treatment of TFTs with nanocrystalline active layer fabricated at low temperature $\left(125^{\circ} \mathrm{C}\right)$ by HWCVD process. The results indicate that changes in the electrical characteristics of the device could be due to temporarily trapped charge in the material. In our future work detailed analysis of the interface states in the transistors by capacitance measurements will be performed.

\section{Acknowledgements}

The authors wish to thank Dr L. Fonseca from Centre Nacional de Microelectrònica for growing the silicon dioxide. This work was supported by the CICYT of the Spanish Government under programme TIC1999-0842.

\section{References}

[1] H. Matsumura, Jpn. J. Appl. Phys. 30 (1991) L1522.

[2] J. Cifre, J. Bertomeu, J. Puigdollers, M.C. Polo, J. Andreu, A. Lloret, Appl. Phys. A 59 (1994) 645.

[3] H. Meiling, R.E.I. Schropp, Appl. Phys. Lett. 69 (8) (1996) 1062.

[4] M.J. Powell, IEEE Trans. Elec. Dev. 36 (12) (1989) 2753.

[5] D. Peiró, PhD Thesis, University of Barcelona (1999).

[6] J. Puigdollers, A. Orpella, D. Dosev, C. Voz, D. Peiró, J. Pallarès, L.F. Marsal, J. Bertomeu, J. Andreu, R. Alcubilla, J. Non-Cryst. Solids 266-269 (2000) 1304.

[7] M.J. Powell, S.C. Deane, W.I. Milne, Appl. Phys. Lett. 60 (2) (1992) 207.

[8] H.C. Slade, M.S. Shur, IEEE Trans Elec. Dev. 45 (7) (1998) 1548-1553.

[9] C. Voz, M. Fonrodona, D. Soler, D. Peiró, J.M. Asensi, J. Bertomeu, J. Andreu, Proceedings of 16th European Photovoltaic Solar Energy Conference, 2000 Glasgow, in print. 